\title{
DEEPLY PENETRATING BANDED ZONAL FLOWS IN THE SOLAR CONVECTION ZONE
}

\author{
R. Howe ${ }^{1}$, J. Christensen-DalsgaArd ${ }^{2}$, F. Hill ${ }^{1}$, R.W. Komm ${ }^{1}$, R.M. LARsen ${ }^{3}$,

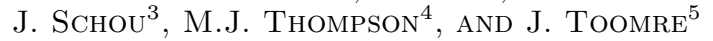 \\ ${ }^{1}$ National Solar Observatory, 950 N. Cherry Avenue, Tucson AZ 85726-6732, USA \\ ${ }^{2}$ Teoretisk Astrofysik Center, Danmarks Grundforskningfond; and \\ Institut for Fysik og Astronomi, Aarhus Universitet, DK-8000 Aarhus C, Denmark \\ ${ }^{3}$ HEPL Annex A201, Stanford University, Stanford CA 94305-4085, USA \\ ${ }^{4}$ Astronomy Unit, Queen Mary \& Westfield College, London E1 4NS, UK \\ ${ }^{5}$ JILA, and Dept. of Astrophysical and Planetary Sciences, University of Colorado, \\ Boulder CO 80309-0440, USA \\ Draft version October 28, 2018
}

\begin{abstract}
Helioseismic observations have detected small temporal variations of the rotation rate below the solar surface corresponding to the so-called 'torsional oscillations' known from Doppler measurements of the surface. These appear as bands of slower and faster than average rotation moving equatorward. Here we establish, using complementary helioseismic observations over four years from the GONG network and from the MDI instrument on board SOHO, that the banded flows are not merely a near-surface phenomenon: rather they extend downward at least $60 \mathrm{Mm}$ (some $8 \%$ of the total solar radius) and thus are evident over a significant fraction of the nearly $200 \mathrm{Mm}$ depth of the solar convection zone.
\end{abstract}

Subject headings: Sun: interior — Sun: oscillations - Sun: rotation

\section{INTRODUCTION}

The intensely turbulent state of the solar convection zone is revealed by the patterns of granulation, mesogranulation and supergranulation evident in its surface layers (e.g., Brummell, Cattaneo \& Toomre 1995). Yet accompanying such turbulent and seemingly chaotic small-scale dynamics are also signs of ordered large-scale behavior. Most notably the solar differential rotation involves a relatively smooth decrease in angular velocity from equator to pole, both in the surface layers (e.g., Snodgrass 1984) and within the convection zone as inferred from helioseismic measurements (e.g., Thompson et al. 1996; Schou et al. 1998a). On the largest scales, the magnetic activity similarly exhibits well-defined rules as the 22-year cycle progresses.

An enticing link between the latitudes of field emergence and small variations in the rotation rate of the surface layers is provided by bands of slightly faster and slower than average zonal flows, called torsional oscillations, that were observed from direct Doppler measurements to migrate towards the equator in a manner similar to the zones of solar activity (e.g., Howard \& LaBonte 1980; Snodgrass, Howard \& Webster 1985; Ulrich 1998). Helioseismic analysis of data from the Michelson Doppler Imager (MDI) instrument (e.g., Scherrer et al. 1995) on the Solar and Heliospheric Observatory (SOHO) spacecraft has confirmed the presence of such bands of weak zonal flow, and their drift towards the equator, for the present solar cycle (Kosovichev \& Schou 1997; Schou et al. 1998a,b; Schou 1999). Although the causal relation between these banded flows and the zones of magnetic activity is still unclear, it is important to understand whether the flows are confined to the layer of rotational shear just below the solar surface. In this letter, we address such questions using two extensive helioseismic data sets, covering slightly over four years, obtained with MDI and with the ground-based Global Os- cillation Network Group (GONG) project (e.g., Harvey et al. 1996). We establish the consistency of the independent determinations of the flow from the two data sets, and infer that the zonal banding signature extends to depths of about $60 \mathrm{Mm}$ (or about $8 \%$ in radius) below the solar surface. Thus these are not superficial features, and provide evidence of ordered rotational responses as the magnetic cycle is progressing. More extensive accounts of such analyses of zonal flows are provided for GONG data by Howe, Komm \& Hill (2000) and for MDI data by Toomre et al. (2000).

\section{OBSERVATIONS AND DATA ANALYSES}

The rotation rate of the solar convection zone has been inferred through inversion of observed rotational splittings of solar $\mathrm{f}$ and $\mathrm{p}$ modes. Two sets of observations have been used. One was obtained by the GONG network over the period 1995 May 7 to 1999 June 26. This set consists of 40 overlapping series of 108 days each, with starting dates 36 days apart. The second set was obtained by MDI and consists of 11 contiguous sets from 1996 May 1 to 1998 June 24 (before control of the spacecraft was lost), one set from 1998 October 23 to 1998 December 21 (after control was reasserted), and four sets from 1999 February 3 to 1999 November 17 . While each of the sets nominally covers 72 days, the ones surrounding the loss of contact are missing a few days.

The dependence of the frequencies on the azimuthal order was represented in terms of an expansion on orthogonal polynomials (Ritzwoller \& Lavely 1991), expressed in terms of the so-called $a$ coefficients $a_{k}(n, l)$, depending on the radial order $n$ and the degree $l$ of the mode, as well as on the order $k$ of the coefficient. The odd coefficients are related to the angular velocity $\Omega(r, \theta)$ (as a function of the 

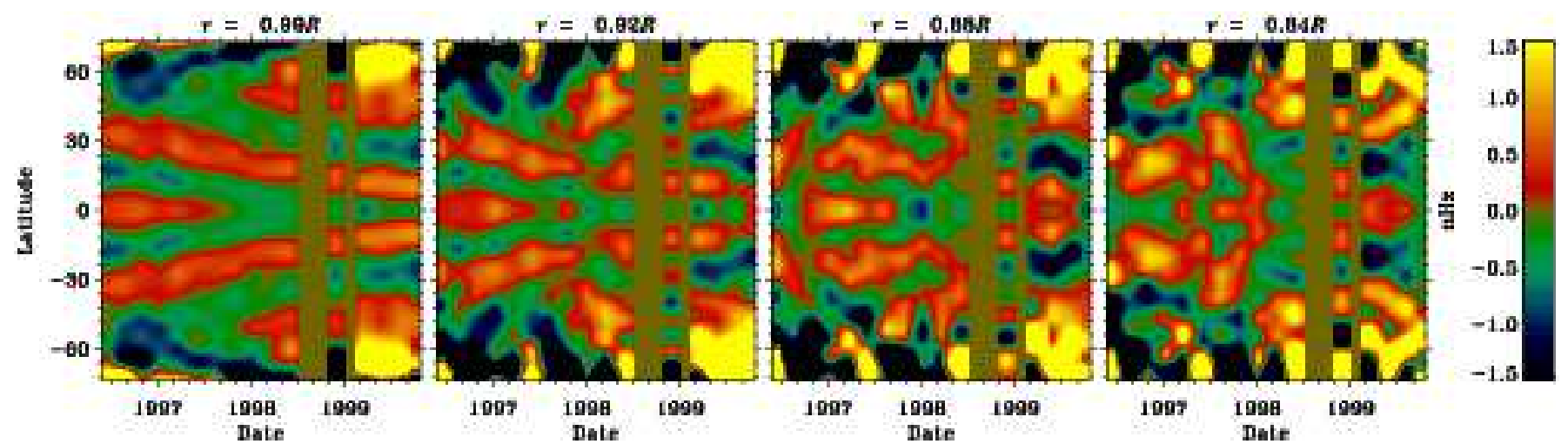

FIG. 1. - Variation of rotation rate with latitude and time from which a temporal average has been subtracted to reveal the migrating banded zonal flows, based on OLA inversion of MDI data for target radii of $0.99 R, 0.92 R, 0.88 R$ and $0.84 R$. The rendition is smoothed in the temporal direction over a window of 72 days, equal to the period of each separate set of observations. Uniform olive-green vertical bands indicate that no data were available in that time period for our analysis. The color bar indicates the dynamic range in nHz of the angular velocity.

distance $r$ to the solar center and the co-latitude $\theta$ ) by

$$
2 \pi a_{2 s+1}(n, l)=\int_{0}^{R} \int_{0}^{\pi} K_{n l s}^{(a)}(r, \theta) \Omega(r, \theta) r \mathrm{~d} r \mathrm{~d} \theta,
$$

where $R$ is the solar radius and the kernels $K_{n l s}^{(a)}$ are assumed known from a solar model. The GONG data comprised around 10,000 coefficients, up to $a_{15}$, for a total of typically $1,200 \mathrm{p}$-mode multiplets $(n, l)$ for $l \leq 150$, whereas the MDI sets contained approximately 30,000 coefficients, up to $a_{35}$, for roughly 1,800 multiplets with $l \leq 300$. The inversions of the relations (1) were carried out by means of two methods, described by Schou et al. (1998a): two-dimensional regularized least-squares fitting (RLS) and two-dimensional subtractive optimally localized averages (OLA).

\section{RESULTS AND DISCUSSION}

The overall features of the inferred rotation profile are very similar to those obtained by Schou et al. (1998a) based on 144 days of MDI data. Here we concentrate on the time-dependent aspects of the dynamics of the upper portions of the convection zone. These are most readily studied by considering departures of the reconstructed rotation rate from its temporal average $\bar{\Omega}(r, \theta)$. Figure 1] shows the evolution of these residuals as a function of latitude at four target depths, using OLA inversion of the MDI data. The inversion is only sensitive to the component of rotation symmetric around the equator; even so, to show more clearly the evolution of the features, we have included both hemispheres in the plots. The residuals show alternating bands of positive and negative zonal velocity, relative to the temporal mean, converging towards the equator with increasing time. The amplitude in these flows is around $1.5 \mathrm{nHz}$, corresponding to velocities of up to around $6 \mathrm{~ms}^{-1}$. The flows are visible, at roughly the same amplitude, in the inversion targeted at $0.92 R$, and faint traces are visible in the inversion targeted at $0.88 R$; we return to the significance of this below.

Although the signal shown in Figure 11 seems strong and coherent, some doubt about its reality may remain. Thus access to the independent GONG dataset is essential, with the additional advantage that it starts almost a year before the MDI data. As a further test, we also apply two different analysis techniques to the set, as shown in Figure 2 .
The GONG and MDI data, where they overlap in time, are essentially consistent at $r=0.99 R$. At $r=0.95 R$ the GONG RLS reconstructions are somewhat noisier so that the subtle signature of the migrating zonal bands is less obvious. The RLS and OLA inversion results for the MDI data agree very well at both depths illustrated.

To provide a more quantitative comparison, Figure 3 shows the GONG and MDI solutions at selected radii and latitudes as a function of time. It is evident that the three sets of results largely agree within their error bars. Also, the variations are highly statistically significant: at the equator the angular velocity decreases uniformly, whereas at latitude $30^{\circ}$, for example, the variation reflects mainly the passage of the band of more rapid rotation. The inversions reveal a considerably different behavior at the two depths at latitude $60^{\circ}$, much as could also be inferred from Figure 2.

The interpretation of any inversion results must take into account their finite resolution, as well as the properties of errors of the inferences. In particular, the solution obtained at a given location contains contributions from $\Omega$ at other points, while error correlation between the solutions at different locations may give the impression of coherent structure where none exists. Quantitative measures of these effects can be obtained from detailed analyses of the inversion (e.g. Schou et al. 1998a; Howe \& Thompson 1996). As an alternative, we have considered artificial data for a number of prescribed rotation laws, with error properties corresponding to those of the solar data. The mode set and errors used correspond to those of a typical MDI set. Superimposed on a smoothly varying flow in the latitudinal direction, the artificial rotation profiles possess a single pair of flows, $10^{\circ}$ wide, and rotating $3 \mathrm{nHz}$ faster than the background level, moving equatorwards at $5^{\circ}$ per sample. There are nine samples in all in each 'time' sequence. Three cases are illustrated in Figure using OLA inversions: in the first, the flows extend from the surface to a depth of $5 \%$ of the solar radius, in the second to a depth of $8 \%$ and in the third to a depth of $20 \%$. For all cases, the branches of the flow are strongly visible at $0.99 R$, and the flows are evident to about the depth to which they are imposed, while disappearing below that depth. The $0.92 R$ case most resembles the solar observations illustrated in Figure 1, whereas the disappearance of the flows in the 
$0.95 R$ case at $0.93 R$ and the visibility of the $0.80 R$ flow at $0.84 R$ are both inconsistent with the solar observations. This evidence, taken together with many other cases which we have tested, strongly suggests that the solar flow structure extends to a depth of at least $0.08 R$ with substantial amplitude, but does not extend much further than $0.10 R$. The latitudinal width of the Sun's banded flows (Fig. 1) is similar to the $10^{\circ}$ width assumed in the artificial data: this is consistent also with the Doppler measurements at the surface (Ulrich, 1998). Comparison of Figures 11 and 4 suggests that the solar flows may be somewhat weaker than the $3 \mathrm{nHz}$ chosen for the artificial case.
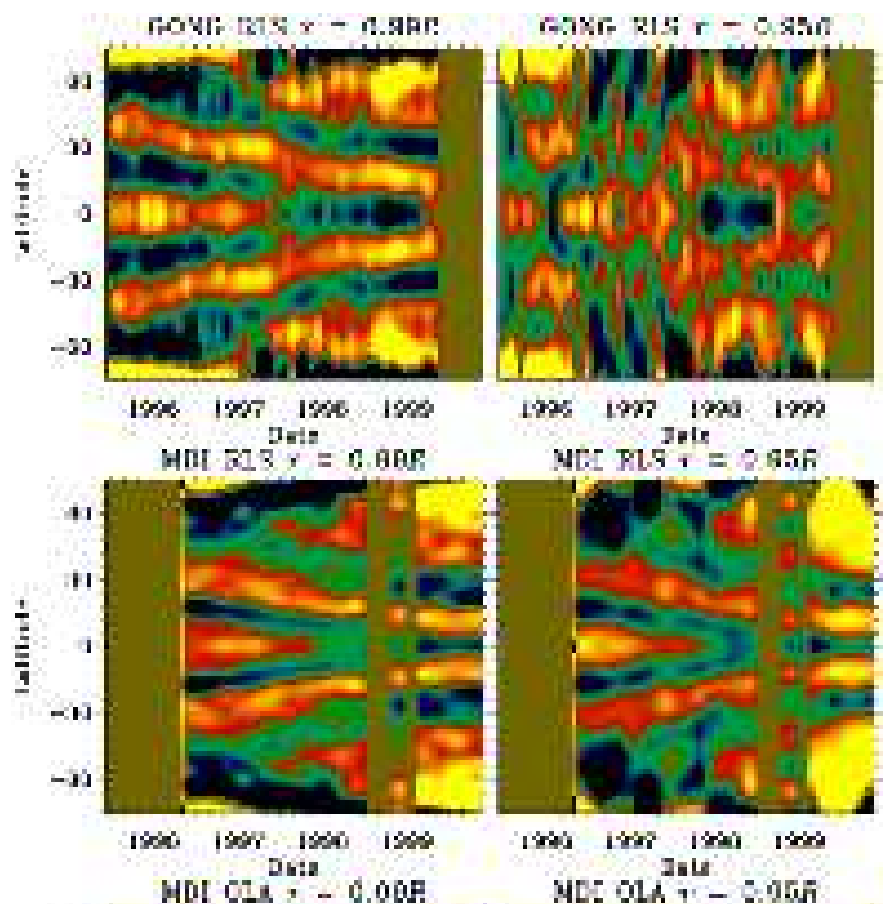
4อ $\mathrm{FLT:}=\operatorname{asc}$
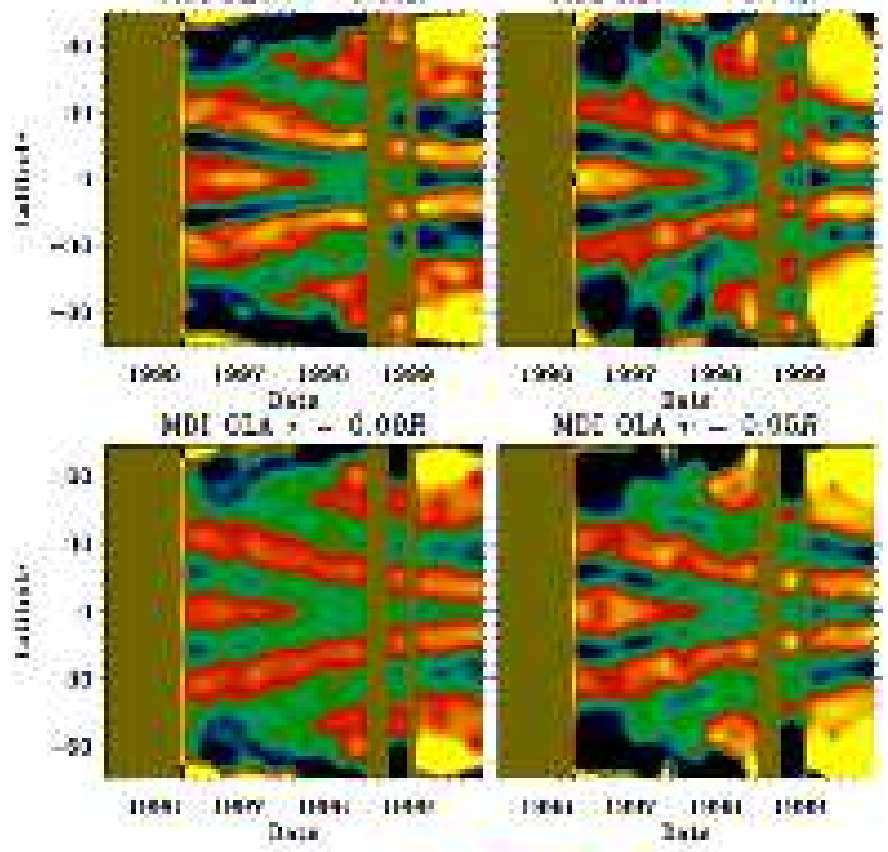
yL: oLh 1 - - gri

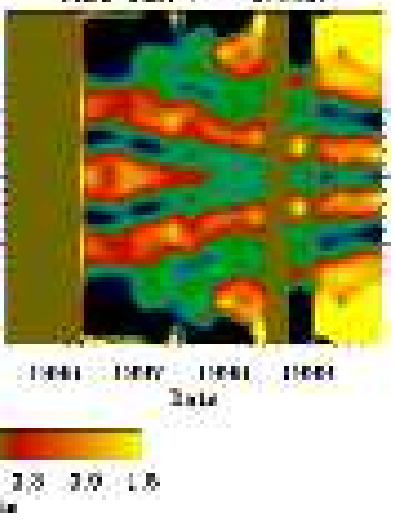

FIG. 2.- Comparison of various inversions of GONG and MDI data showing evolution of residual rotation rate (cf. Fig. 1) at target radii $0.99 R$ (left column) and $0.95 R$ (right column). From the top, the three rows show reconstructions with RLS using GONG data, with RLS using MDI data, and with OLA using MDI data.

At the highest latitudes, Figures 1 and 2 show more dynamical variations than at lower latitudes. This is likely related to the lesser moment of inertia associated with the polar regions. There may be evidence for the formation of a new band of rapid rotation tending towards lower latitudes; the further evolution of this feature should be followed over the coming years.
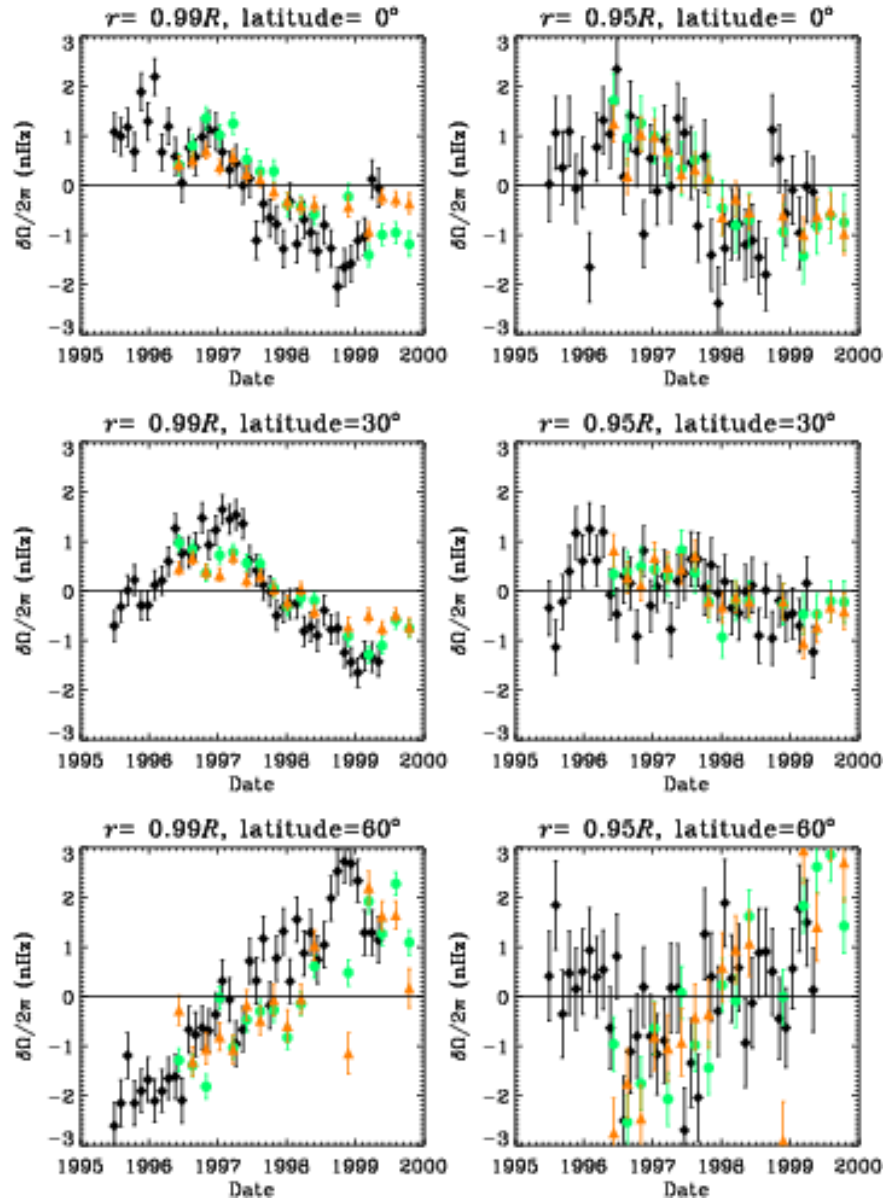

FIG. 3. - Evolution with time in the residual rotation rate at target radii $0.99 R$ and $0.95 R$ and latitudes $0^{\circ}, 30^{\circ}$, and $60^{\circ}$. Diamonds (black) show results of RLS inversion applied to GONG data, whereas circles (green) and triangles (orange) are for RLS and OLA inversions on MDI data. The error bars show one standard deviation of the inference, as determined from the errors in the observed data.

\section{CONCLUSIONS}

Analysis of extended series of GONG and MDI data has revealed coherent banded flow structures in the solar convection zone. These correspond to the torsional oscillations detected in direct Doppler observations of the solar surface. We have demonstrated that the flows are likely to extend to a depth of at least $60 \mathrm{Mm}$, a substantial fraction of the total $200 \mathrm{Mm}$ depth of the convection zone, and considerably more than the depth (about $35 \mathrm{Mm}$ ) at which the rotation rate attains its maximum in the subsurface radial shear layer at low latitudes (cf. Schou et al. 1998a). In addition, there appear to be other systematic variations with time of the residual rotation rate, with different signatures at low and high latitudes (cf. Fig. 3).

Inversions of global oscillation frequency splittings sample the component of rotation symmetric around the equator. The actual flows will exhibit some level of asymmetry which will depend on the time scale used in the analysis. Indeed, local analyses by means of the time-distance and ring-diagram techniques (Giles, Duvall, \& Scherrer 1998; Haber et al. 2000) have shown features similar to those found here, but with clear differences between the two hemispheres. 


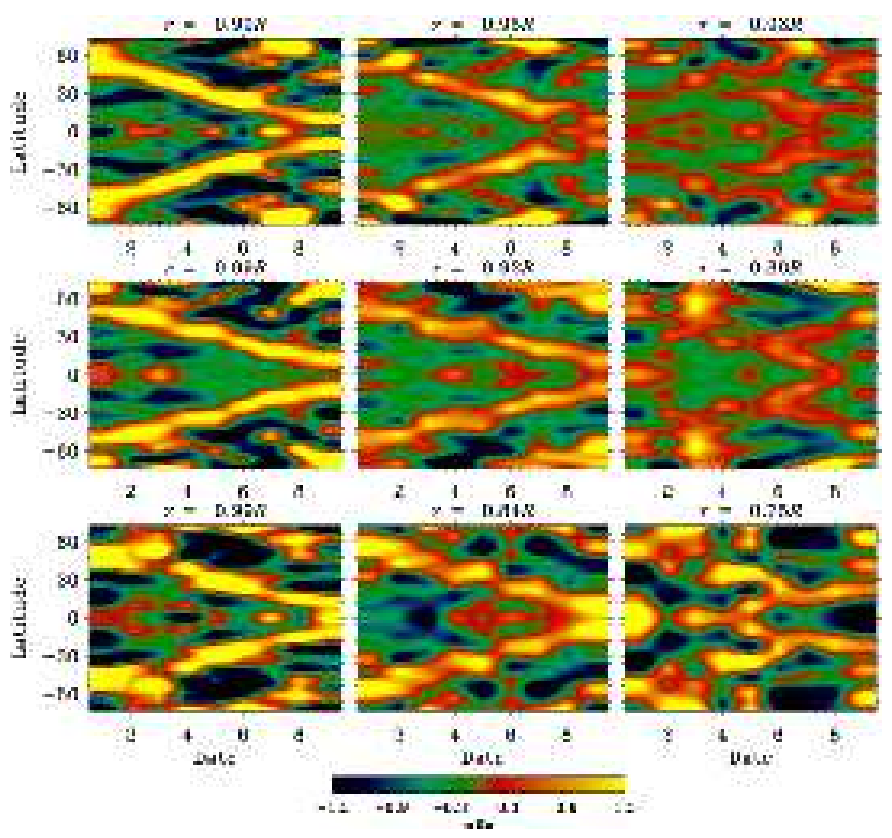

FIG. 4.- Sample artificial data tests to study residual rotation rate inferred from OLA inversions at various selected radii. The imposed flow used in the calculation of the frequency splittings extends from the surface to $0.95 R$ in the top row, to $0.92 R$ in the middle row, and to $0.80 R$ in the bottom row.

The link between the evolving latitudinal positioning of the faster zonal bands and of the sites of sunspot emergence suggest that the dynamics are related, yet how this is accomplished is uncertain. The strong magnetic fields most likely originate from deep within the Sun, probably formed by dynamo action near the base of the convection zone (e.g., Spiegel \& Zahn 1992; Parker 1993; Weiss 1994; Charbonneau \& MacGregor 1997). Field bundles ascending from this region through the convection zone, before erupting into the atmosphere as large-scale magnetic loops, could well lead to significant perturbations in velocity and thermal fields there. This is likely to be accompanied by some redistribution of angular momentum, given that the magnetic structures will attempt to conserve their original angular momentum (e.g., Brummell, Cattaneo \& Toomre 1995). The coupling of a highly tur- bulent medium with ascending magnetic structures, and their mutual feedbacks, have not yet been assessed in recent flux-tube models. Global simulations of turbulent convection in rotating spherical shells (e.g., Elliott et al. 2000; Miesch et al. 2000; Miesch 2000) to study the resulting differential rotation have revealed intrinsic variability in zonal flows over intervals of several rotation periods, some of which may be inertial oscillations (e.g., Gunther \& Gilman 1985), but such modelling has not included largescale magnetic fields. Obtaining propagating bands and time scales of variation of order the solar cycle seems problematic unless there is some selective coupling to magnetic processes. Adding to the puzzle is that the evolving zonal bands are present at the higher latitudes even before the prominent large-scale magnetic eruptions begin (e.g. Ulrich 1998), as within this cycle. Continued helioseismic observations as this magnetic cycle is proceeding may help to provide clues about such aspects of solar internal dynamics, for we now have the ability to probe hitherto unseen flows well below the solar surface.

\section{ACKNOWLEDGEMENTS}

This work utilizes data obtained by the GONG project, managed by the National Solar Observatory, a Division of the National Optical Astronomy Observatories, which is operated by AURA, Inc. under a cooperative agreement with NSF. The data were acquired by instruments operated by the Big Bear Solar Observatory, High Altitude Observatory, Learmonth Solar Observatory, Udaipur Solar Observatory, Instituto de Astrofísico de Canarias, and Cerro Tololo Interamerican Observatory. The Solar Oscillations Investigation (SOI) involving MDI is supported by NASA grant NAG 5-3077 to Stanford University. SOHO is a mission of international cooperation between ESA and NASA. RWK, and RH in part, were supported by NASA contract S-92698-F. JC-D was supported by the Danish National Research Foundation through the establishment of the Theoretical Astrophysics Center. MJT was supported in part by the UK Particle Physics \& Astronomy Research Council. JT was supported in part by NASA through grants NAG 5-7996 and NAG 5-8133, and by NSF through grant ATM-9731676.

\section{REFERENCES}

Brummell, N., Cattaneo, F., \& Toomre, J. 1995, Science, 269, 1370

Charbonneau, P., \& MacGregor, K.B. 1997, ApJ, 486, 502

Elliott, J.R., Miesch, M.S., \& Toomre, J. 2000, ApJ, in press

Giles, P. M., Duvall, T. L. Jr., \& Scherrer, P. H. 1998, in Proc. SOHO 6/GONG 98 Workshop, Structure and Dynamics of the Interior of the Sun and Sun-like Stars, ed. S.G. Korzennik, \& A. Wilson (ESA SP-418), (Noordwijk: ESA), 775

Gunther, D.B., \& Gilman, P.A. 1985, ApJ, 295, 195

Haber, D.A., Hindman, B.W., Toomre, J., Bogart, R.S., Thompson, M.J., \& Hill, F. 2000, Solar Phys., in press

Harvey, J.W., et al. 1996, Science, 272, 1284

Howard, R., \& LaBonte, B. J. 1980, ApJ, 239, L33

Howe, R., Komm, R., \& Hill, F. 2000, Solar Phys., in press

Howe, R., \& Thompson, M.J. 1996, MNRAS, 281, 1385

Kosovichev, A.G., \& Schou, J. 1997, ApJ, 482, L207

Miesch, M.S. 2000, Solar Phys., in press

Miesch, M.S., Elliott, J.R., Toomre, J., Clune, T.L., Glatzmaier, G.A., \& Gilman, P.A. 2000, ApJ, in press

Parker, E.N. 1993, ApJ, 408, 707
Ritzwoller, M.H., \& Lavely, E.M. 1991, ApJ, 369, 557

Scherrer, P.H., et al. 1995, Solar Phys., 162, 129

Schou, J. 1999, ApJ, 523, L181

Schou, J., et al. 1998a, ApJ, 505, 390

Schou, J., et al. 1998b, in Proc. IAU Symp. 185: New Eyes to See Inside the Sun and Stars, ed. F.-L. Deubner, J. ChristensenDalsgaard, \& D.W. Kurtz (Dordrecht: Kluwer), 141

Snodgrass, H.B., Howard, R.F., \& Webster, L. 1985, Solar Phys., 95, 221

Spiegel, E.A., \& Zahn, J.-P. 1992, A\&A, 265, 106

Thompson, M.J., et al. 1996, Science, 272, 1300

Toomre, J., Christensen-Dalsgaard, J., Howe, R., Larsen, R.M., Schou, J., \& Thompson, M.J. 2000, Solar Phys., in press

Ulrich, R.K. 1998, in Proc. SOHO 6/GONG 98 Workshop, Structure and Dynamics of the Interior of the Sun and Sun-like Stars, ed. S.G. Korzennik, \& A. Wilson (ESA SP-418), (Noordwijk: ESA), 851

Weiss, N.O. 1994, in Lectures on Solar and Planetary Dynamos, ed. M.R.E. Proctor, \& A.D. Gilbert (Cambridge: Cambridge), 59 